\title{
Políticas públicas chinas: bibliografía existente, instituciones transicionales y centralización-descentralización
}

\section{Chinese public policies: existing bibliography, transitional institutions and} centralization-decentralization

Martín Rozengardt ${ }^{1}$

\section{Resumen:}

El área de estudios referida a políticas públicas chinas resguarda una vasta bibliografía, que dista de estar organizada o sistematizada. El presente artículo recopila dicho material académico, específicamente enfocado en la producción de políticas públicas desde 1978 hasta la actualidad. Por otra parte, introduce las principales discusiones en torno al tema, tomando como punto de partida las definiciones de Xue Lan y Wang Shaoguang, que destacan el rol de la dinámica “centralización-descentralización” y la importancia de una visión longitudinal del proceso.

Con este análisis, el documento desarrolla una posible tipología de diversos modelos de producción de políticas públicas y plantea la posibilidad de su connivencia, según los distintos momentos de la reforma del Estado en China.

Palabras Clave: China, Políticas públicas, Instituciones transnacionales, Centralizacióndescentralización.

\footnotetext{
${ }^{1}$ Maestrando del programa de Estudios chinos de la Universidad de Shanghái, China; especializado en las relaciones entre Argentina y Asia, con interés particular por la investigación sobre la gestión de políticas públicas en China.
}

Orientando. Temas de Asia Oriental. Sociedad, Cultura y Economía. Revista editada por el Centro de Estudios China-Veracruz de la Universidad Veracruzana, México (Centro de Estudios APEC) / año g / número 17 / 


\begin{abstract}
:
The area of studies related to Chinese public policies has a wide bibliography, which is far from being organized or systematized. This article compiles said academic material, specifically focused on the production of public policies from 1978 to the present. On the other hand, it introduces the main discussions around the subject, taking as a starting point the definitions of Xue Lan and Wang Shaoguang, which highlight the role of the "centralization-decentralization" dynamic and the importance of a longitudinal view of the process.
\end{abstract}

With this analysis, the document develops a possible typology of various models of public policy production and raises the possibility of their collusion, according to the different moments of the state reform in China.

Keywords: China, Public policies, Transnational institutions, Centralization-decentralization.

Orientando. Temas de Asia Oriental. Sociedad, Cultura y Economía. Revista editada por el Centro de Estudios China-Veracruz de la Universidad Veracruzana, México (Centro de Estudios APEC) / año 9 / número 17 / 


\section{1. "Ponerse al día"}

Una de las frases emblemáticas de los años posteriores a la reforma de apertura y modernización en China, fue la expresión de Deng Xiaoping referida a la necesidad de "ponerse al día” -en chino “补课”. Esta enmienda se expresó en el campo académico con el nombramiento de Fei Xiaotong ${ }^{2}$ como primer director del Instituto de Sociología de la Academia China de Ciencias Sociales; pero, desde una óptica integral, esta cita reflejaba el proceso de transformación estructural que comenzó a atravesar la nación asiática en 1978.

Este proceso de cambio, ejecutado por el Estado de la República Popular China (RPCh), bajo la dirección del Partido Comunista Chino (PCC), ha sido reflexionado con mayor frecuencia desde el ámbito económico; con un énfasis especial en la transición suscitada de una economía planificada a una de mercado, que no dejó de generar un crecimiento sostenido. (Qian, 2002; Kroeber; 2016; Xu, 2011).

Sin embargo, este proceso fue mucho más complejo: desdibujó grandes brechas entre diversos sectores -interior/costa, rural/urbano, ciudades ricas/provincias pobres, entre otros- ${ }^{-}$, pero eficaces medidas contra la pobreza ${ }^{3}$; también generó el desarrollo de entidades privadas, fortaleció el control del Estado sobre recursos estratégicos y puso en el eje mediático una alianza con Estados Unidos, con una hipótesis sobre una posible dirección multipolar del globo. (Dussel, 2015) De igual forma, presentó diferencias con el maoísmo y la continuidad del rol hegemónico del PCC; solo por enumerar algunos ejemplos de la inmensidad de matices involucrados en los períodos de transformación de China.

Este impulso por "ponerse al día" se combinó con el proceso de apertura y dio a lugar a un mayor diálogo entre académicos e intelectuales chinos y del resto del mundo,

\footnotetext{
${ }^{2}$ Es considerado uno de los fundadores de la sociología y la antropología de China. En 1939, regresó al país después de estudiar con Malinowski; para ese entonces, las ciencias sociales estaban pasando por un proceso de institucionalización y desarrollo. A partir de 1978 fue el encargado impulsar la sociología a nivel nacional. 3 El coeficiente de Gini aumentó en las áreas urbanas de 0.16, en 1978, a 0.36, en 2007, y de 0.22 a 0.38 para las áreas rurales en el mismo período. La pobreza disminuyó en un 90\%, desde fines de la década del 70 hasta el 2007. (Li, 2011)
}

Orientando. Temas de Asia Oriental. Sociedad, Cultura y Economía. Revista editada por el Centro de Estudios China-Veracruz de la Universidad Veracruzana, México (Centro de Estudios APEC) / año 9 / número 17 / 
configurando nuevas dinámicas para el desarrollo de investigaciones. Por ello, las disciplinas científicas, en continua correspondencia con las demandas e inquietudes del Estado, aumentaron su producción y especificación.

En tal sentido, este artículo tiene como objetivo principal describir y sistematizar la literatura existente sobre el estudio de políticas públicas chinas, priorizando a los autores locales, en tanto casos posibles de especificación de la sociología china posteriores a 1978.

\section{Preliminares}

En un texto ya clásico de la época de las reformas radicales de la década de los 80 , Theda Skocpol distingue dos estrategias analíticas alternativas para el gran campo que estudia el papel del Estado. La primera considera a los Estados como "organizaciones mediante las cuales los colectivos de funcionarios pueden perseguir objetivos característicos, alcanzándolos con mayor o menor eficacia según los recursos estatales disponibles en relación con los marcos sociales.” (Skocpol, 1985)

La segunda los describe como "configuraciones de organización y acción que influyen en los significados y métodos de la política para todos los grupos y clases de la Sociedad". (Íbid, p. 41) A la luz del debate disciplinar, estas definiciones tienen un gran potencial heurístico para comprender el caso en China.

Si la primera definición acepta relativos grados de autonomía, la segunda incorpora elementos relacionales. Una tercera posibilidad podría indagar sobre cómo las estructuras, fuerzas sociales y los actores sociales no estatales determinan o condicionan a dicho colectivo de funcionarios. Todas ellas transitan sobre los debates entre Estado y sociedad y sus variantes.

Orientando. Temas de Asia Oriental. Sociedad, Cultura y Economía. Revista editada por el Centro de Estudios China-Veracruz de la Universidad Veracruzana, México (Centro de Estudios APEC) / año g / número 17 / 
Sin embargo, a pesar de que los ejercicios analíticos recortan el análisis en una u otra estrategia, aparecen de manera interrelacionada. Hechas las salvedades correspondientes, el presente artículo se enmarca -aunque no de manera excluyente- en la primera y la tercera vertiente de análisis.

La propuesta de Skocpol plantea preguntas acerca de que el Estado "regrese al primer plano". No obstante, estos debates pueden remontarse a discusiones de orden filosófico. En ese sentido, hay que mencionar a los múltiples autores que abordaron este tema en la modernidad: Durkheim, Marx, Weber, Gramsci, Jessop, Anderson, Mann, Tarcus, Giddens y Bourdieu, por nombrar algunos. Así mismo, desde muchas otras regiones del mundo se ha reflexionado sobre esta área de estudio.

Pero en América Latina y el Caribe, China y tantas de las otras regiones, países y territorios delimitados, la producción académica tiene menos posibilidades de distribución a escala global. Por ejemplo, en Bolivia, García Linera define al Estado en tanto "síntesis colectiva de la sociedad con liderazgo interno que utiliza sus mecanismos - los monopolios coercitivos, tributarios y propietarios- para cierto tipo de iniciativas; es decir, es un pedazo de la sociedad, jerarquizado internamente y con capacidad de decisión”. (García, 2009)

Desde un marco más cercano a Webber, O’Donnell lo define como "un conjunto de instituciones y de relaciones sociales - la mayor parte de estas sancionadas por el sistema legal- que normalmente penetra y controla el territorio y a los habitantes que pretende delimitar geográficamente”. (O’Donnell, S.F.).

Debido a que este escrito se enfoca en China, se establece como objetivo partir de las nociones de Estado propuestas por autores locales, como sucederá en el segundo apartado. Porque, como explica Zavaleta Mercado (1990), “el Estado debe estudiarse como situación concreta, como agregación histórica y como particularidad".

Las investigaciones que toman a China como caso de estudio deben traducir con cautela la bibliografía existente, debido a que enfrentan el riesgo de generar concepciones orientalistas. Para sortear obstáculos epistemológicos, estas producciones académicas deben

Orientando. Temas de Asia Oriental. Sociedad, Cultura y Economía. Revista editada por el Centro de Estudios China-Veracruz de la Universidad Veracruzana, México (Centro de Estudios APEC) / año g / número 17 / 
figurar con cierta jerarquía frente a las voces provenientes de otras partes del mundo y sus marcos conceptuales.

Uno de los aportes centrales en este marco es el de Ma y Lin (2012), cuyo artículo presenta una gran cantidad de investigaciones locales sobre políticas públicas chinas. De igual forma, existen otras investigaciones realizadas fuera de China; destaca un consenso académico acerca del libro Bureaucracy, Politics, and Decision Making in Post-Mao China Studies On China, de Liberthal y Lampton y su concepto de "autoritarismo fragmentado". Incluso, Ma y Lin aclaran que hubo un período de "silencio" en la producción académica local respecto al estudio de políticas públicas ${ }^{4}$ y que la mayor profundización al respecto comenzó en otras partes del mundo.

De acuerdo con los mismos autores, Wang Shaoguang y Xue Lan fueron dos de los principales académicos en el estudio de las políticas públicas. El primero propuso un modelo en una publicación titulada "Changing Models of China's Policy Agenda Setting”, en 2008. 5 El segundo explicó la relación entre el tipo de políticas públicas implementadas y la dinámica gobierno central-gobierno local-mercado, en el artículo "When gradualism might fail? Politics of Policy Experiment in China", en 2012. ${ }^{6}$

Además de estas dos publicaciones clave, dada su referencia explícita a la producción de políticas públicas, ambos autores tienen en su haber una variada bibliografía traducida al inglés, donde plantean muchas de las interrogantes fundamentales. En esta cronología, abren preguntas acerca del rol del Estado -Wang, 1994 y 1995 y Xue, 2012-, de las capacidades

\footnotetext{
4 El texto refiere explícitamente al policy making process. Para simplificar sin dejar de traducir, se hace referencia al estudio de políticas públicas, a pesar de no ser exactamente lo mismo. Por otro lado, se utiliza, a modo de simplificación, el término "producción de políticas públicas" para aludir al proceso que incluye las etapas de emergencia, realización, revisión, ejecución e implementación de políticas públicas.

${ }^{5}$ Wang Shaoguang. (2008). “Changing Models of China's. Policy Agenda Setting”. In Modern China(34), N. 1. Sage Publications.

${ }^{6}$ Mei Ciqi, Liu Zhilin \& Xue Lan. (2012). "When gradualism might fail? Politics of Policy Experiment in China". School of Public Policy \& Management, Tsinghua University, Beijing, China. Paper Presented at the 22rd International Political Science Association World Congress. Madrid, Spain, July 8-12.
}

Orientando. Temas de Asia Oriental. Sociedad, Cultura y Economía. Revista editada por el Centro de Estudios China-Veracruz de la Universidad Veracruzana, México (Centro de Estudios APEC) / año g / número 17 / 
estatales -Wang, 1991 y 2000-, y también de los actores y las instituciones del mercado -Wang, 1994 y 2010 y Xue, 2012.

Por otra parte, algunas cuestiones atraviesan este recorrido, relativas a la teoría social sobre China analizada a través de autores locales. Por ejemplo, respecto a los debates entre quienes afirman un aumento en la participación de actores externos al sistema estatal, en contraposición a un mayor control por parte de la cúpula dirigente -especialmente a partir del 2003. También subyace el esfuerzo de algunos autores por sistematizar las fases o los momentos del mismo proceso.

Además, se articulan dos ejes con un lugar significativo en las investigaciones de Wang y Xue: el primero es la dinámica descentralización-centralización -que podría ser un campo de estudio en sí misma-, sustentada en los distintos lugares que ocupan los gobiernos locales en relación con el gobierno central y el devenir histórico de dicha relación. En esta línea, las distintas características que asumieron según el momento histórico, trazaron las múltiples formas y momentos de producción de políticas públicas en China.

El segundo considera este proceso como algo que cambia con el tiempo; es decir, que posee un sentido histórico y contradictorio. (Foucault, 1977) Algunas de las formas en que se conceptualizó esa peculiaridad en el caso chino son expuestas por Liping (2008), Xue (2012) y Heilmann (2007): transición, gradualismo y experimentación. Para la mejor comprensión de esta característica se introducirá el concepto de "instituciones transicionales", abordado por Qian (2002), un elemento muy importante del proceso de reforma en ese país, que ha tenido poca difusión.

En síntesis, con el aporte de Ma y Lin como punto de partida, este artículo profundiza las ideas de algunos intelectuales en dicho campo de estudio - como Wang o Xue, entre otros-; también actualiza la bibliografía existente, incluyendo como contrapunto investigaciones de autores en otras partes del mundo. Por último, hace hincapié en la relación de las políticas públicas con algunos fundamentos de la reforma del Estado, tales como la dinámica 
centralización-descentralización en China a partir de la reforma de 1978 y el rol de las instituciones transicionales.

\section{De Wang a Xue:}

\section{hacia el análisis integral de las políticas públicas}

Esta sección recopila la literatura existente en este terreno de investigación. A modo de introducción, se pretende hacer dialogar a los dos intelectuales chinos que lideraron la puesta al día de la sociología en el área de estudios acotada: Wang Shaoguan y Xue Lan.

\subsection{Wang Shaoguan: apuntes imprescindibles}

En su artículo "Changing Models of China's Policy Agenda Setting”, mencionado previamente, Wang propone seis formas posibles de conformación de la agenda de políticas públicas en China, desde el maoísmo hasta el año de su publicación; todavía bajo la gestión de Hu Jintao - del 2003 al 2013. Lo que determina el tipo de modelo es la relación entre el actor que propone la política -puede tratarse de líderes del Gobierno, consultores o la ciudadaníay el grado de participación del público. En síntesis, los seis modelos presentan las siguientes condiciones:

1. si quienes inician la política son líderes del Gobierno y la participación es baja, el modelo es a puerta cerrada;

2. cuando los iniciadores son los mismos, pero motivan una alta participación de sectores públicos, el modelo es de movilización;

3. si la política surge de consultores y la participación es baja, es decir que nace de un diálogo cerrado con quienes ejecutarán la política, es de acceso interno; 
4. si los consultores se apoyan en la opinión pública para dar fuerza a su propuesta, el modelo es de alcance;

5. si es la ciudadanía es quien impone la agenda de la política, los modelos pueden ser de acceso externo, porque emerge de un grupo de ciudadanos y no hay altos grados de participación o de presión popular, cuando la demanda es masiva.

Todos los modelos, aclara Wang (2008), conviven y deben ser analizados según cada política. Aun así, él observa una tendencia a una mayor incorporación de la ciudadanía como iniciadora, sobre todo a partir de fines de la década de los 90. En ese momento, el modelo de presión popular era el menos observable, pero algunos acontecimientos -como el aumento de las brechas regionales y de ingreso o el deterioro del medioambiente- motivaron su ascenso. También existieron otros procesos concomitantes: el incremento del asociativismo -particularmente en organizaciones sin fines de lucro-, el nuevo rol de los medios de comunicación, al ascenso de Internet y un aumento en la heterogeneidad de la sociedad china, que tuvo como efecto la diferenciación de intereses particulares.

Estos tipos ideales de formación de la agenda pública describen un recorte muy específico acerca de los actores que intervienen en la emergencia de distintas políticas. El Estado aparece representado de forma monolítica en la toma de decisiones, sin diferenciar entre los gobiernos locales y los centrales, ni su relación o las tensiones políticas internas. La tipología tampoco parece considerar el hecho de que actores externos influyan de manera sustantiva.

Wang tiene una vasta e interesante producción académica respecto a la simplificación del Estado. Por ejemplo, contrapone dos formas de conceptualizarlo: como una abstracción simplificada o como un complejo concreto de componentes dispares:

El problema analítico es que el Estado abarca dos significados. Por un lado, puede entenderse como una abstracción que trasciende todos sus componentes. En tanto abstracción, el concepto de Estado es útil en pos de apropiarse de una realidad compleja y explicarla. Cuando discutimos las relaciones entre el Estado y la sociedad,

Orientando. Temas de Asia Oriental. Sociedad, Cultura y Economía. Revista editada por el Centro de Estudios China-Veracruz de la Universidad Veracruzana, México (Centro de Estudios APEC) / año g / número 17 / 
usualmente usamos el concepto en este sentido. Por otro lado, el Estado puede ser tratado como un complejo concreto de componentes dispares. El reconocimiento de la disparidad de los elementos nos permite comprender la incoherencia, las contradicciones, los dilemas y las tensiones dentro del Estado. Usamos el concepto de Estado en este sentido cuando discutimos temas como la política burocrática y las relaciones centro-gobierno local. (Wang, 1991)

Esta noción más compleja de Estado aparece en muchos de sus artículos traducidos al inglés. Por ejemplo, es utilizada para explorar los efectos del proceso de descentralización ocurrido en la década de los 80, en tanto fragmentación e involución de aparato estatal; eje reflexionado a través del estudio del sistema de pensiones, en 1995, o del estudio de las políticas fiscales, en 1991 y 1995.

Por otra parte, el publicado por Wang en 1991, bajo el título "From Revolution to Involution: State Capacity, Local Power, and [Un]governability in China", es relevante por dos motivos: primero, porque se publica en un momento de efervescente discusión acerca del rol del Estado y de grandes reacomodos. Segundo, porque introduce y entrecruza los dos ejes de interés de este artículo: la dinámica centralización-descentralización y la relación Estadodesarrollo histórico, en este caso bajo el concepto de transición.

Una de las definiciones elementales de ese artículo es la de "capacidad estatal", entendida como "la capacidad del gobierno para movilizar y dirigir los recursos de la sociedad hacia los fines elegidos por el liderazgo político". (Íbid.) El autor propone analizarla en forma longitudinal; es decir, comparándola con los períodos anteriores. Para hacerlo, toma la capacidad extractiva como indicador; además, las políticas fiscales son centrales para su análisis, hecho que da cuenta de una mirada aguda en la coyuntura china. Tres años después de la publicación del artículo, el gobierno central chino ejecutó la gran reforma fiscal de 1994.

En tanto, el ya citado artículo de Skocpol, también utiliza el concepto de capacidad estatal. Por eso, el documento de Wang puede ser pensado en relación a la ya mencionada

Orientando. Temas de Asia Oriental. Sociedad, Cultura y Economía. Revista editada por el Centro de Estudios China-Veracruz de la Universidad Veracruzana, México (Centro de Estudios APEC) / año g / número 17 / 
primera estrategia analítica para los estudios sobre el Estado. En el caso de la socióloga estadounidense, la definición está ligada a la de autonomía relativa y hace referencia a que los objetivos perseguidos por los gobiernos no son "un simple reflejo de las demandas o los intereses de grupos o clases sociales" y que "la explicación de las capacidades del Estado está estrechamente relacionada con la explicación de la fijación de objetivos autónomos por parte de los Estados". (Skocpol, 1985)

Hay una diferencia importante entre ambas concepciones. Mientras que para la última las capacidades estatales están relacionadas con la autonomía del Estado frente a fuerzas y actores externos, el sociólogo chino piensa en la autonomía necesaria para que el gobierno central despliegue toda su capacidad frente a los gobiernos locales.

A pesar de que el proceso de descentralización, ejecutado en la década de los 80, convirtió a los gobiernos locales en uno de los motores de la expansión del mercado, generó también la fragmentación del Estado chino al debilitar la capacidad del gobierno central a finales de esa década. Este indicador fue tan significativo que se hablaba de un "segundo presupuesto"7, en referencia a los mecanismos de los gobiernos locales para manejar sus intereses particulares. (Whiting, 2000)

Sin embargo, no debe entenderse a Wang como un defensor de la centralidad estatal, sino más bien de un "grado normal de descentralización". En su análisis sobre la reforma en el sistema de pensiones, publicado en 1995, el académico plantea que a pesar de que la fragmentación atenta contra la ejecución de una política pública armónica, la descentralización también genera la posibilidad de experimentación -en tanto proceso de emergencia de políticas- y ayuda a que las reformas se desarrollen de manera más veloz.

Para Wang, en vez de hablarse de Estado, es mejor referirse a "sistema estatal"; compuesto por un gobierno central y una determinada cantidad de gobiernos locales. El

\footnotetext{
7 “'....] en 1987, la suma total de ingresos presupuestarios y extrapresupuestarios y tanpai ascendió a 460 mil millones de yuanes, lo que representa el 50,3\% del ingreso nacional de ese año. En comparación con otros países, la proporción era muy alta. Pero, de 460 mil millones, el gobierno central pudo controlar solo el 26\%, o 120 mil millones." (Wang, 1991)
}

Orientando. Temas de Asia Oriental. Sociedad, Cultura y Economía. Revista editada por el Centro de Estudios China-Veracruz de la Universidad Veracruzana, México (Centro de Estudios APEC) / año 9 / número 17 / 
primero le otorga al sistema estatal la efectividad y los segundos la horizontalidad o extensión. De todas maneras, advierte, la efectividad de la acción del centro no puede realizarse sin que los actores locales actúen como correa de transmisión, minimizando las desviaciones de las políticas centrales.

Otro artículo medular en este tema, es el ya mencionado análisis sobre los cambios en las políticas de acceso a la salud, publicado en 2010. En este caso, asume mayor importancia la presencia del mercado, en tanto fuerza que guio, desde la reforma hasta el 2002, los cambios en esta área. Por tanto, dicho artículo explica que las políticas orientadas al mercado durante las décadas de los 80 y los 90 generaron brechas en el acceso a la salud; pero que, luego del fin del mandato de Jiang Zemin, políticas con mayor presencia estatal inauguraron un período de mayor inclusión, un contra movimiento en defensa de la sociedad.

Hasta aquí, los párrafos anteriores recuperaron el pensamiento de Wang Shaoguang: en un primer momento, presentaron su modelo sobre la construcción de la agenda de políticas públicas, luego una serie de análisis más complejos sobre los efectos de la descentralización en las capacidades del sistema estatal chino y el rol del gobierno. No obstante, más allá de los recién nombrados análisis, sus investigaciones acerca de la reforma de pensiones o de las políticas de acceso a la salud y su modelo de agenda de políticas públicas no muestra la complejidad que sí mostraron los otros análisis.

Wang comprende que la producción políticas públicas en China depende de variables complejas -expansión del mercado, dinámica centralización-descentralización, experimentación, etc.- , pero no las relaciona en un artículo global. Por el contrario, Xue Lan sí realiza esta tarea, a través de su grupo de estudios sobre políticas públicas de la Universidad de Tsinghua.

\subsection{Xue Lan: síntesis de conocimientos}

Orientando. Temas de Asia Oriental. Sociedad, Cultura y Economía. Revista editada por el Centro de Estudios China-Veracruz de la Universidad Veracruzana, México (Centro de Estudios APEC) / año g / número 17 / 
En el artículo que este autor escribe junto a Mei Ciqi y Liu Zhilin (2012), pone a prueba el modelo de gradualismo basado en experimentación como uno de los más exitosos en el proceso de reforma de China, desde 1978. Uno de los autores que sintetizó este modelo fue el politólogo alemán Sebastian Heilmann (2007):

En China la experimentación en políticas no implica un proceso desinteresado de prueba y error o una espontánea difusión de políticas. Es una actividad intencional y coordinada, orientada a producir nuevas opciones de políticas que, a su vez, estén inmersas en el proceso oficial de producción de políticas y que luego se replican a gran escala o se incorporan formalmente en la legislación nacional.

Esta forma que adquiere la praxis estatal, requiere de ciertas condiciones propias del caso chino. Una de las más importantes, como ya se mencionó, es la dinámica centralizacióndescentralización, en tanto fuerza productiva e innovadora. A su vez, se contrapone con otros modelos, como el de arriba hacia abajo -en que el Gobierno central implanta la política- o el de abajo hacia arriba, impulsado por las presiones de otros actores de la sociedad.

Sin embargo, Mei, Liu y Xue ponen paños fríos a cerrar un modelo único; este es uno de los puntos más interesantes de su artículo, pues apuntan que no existen los absolutos en cuanto a políticas públicas, pues cada caso y cada política implementada contiene particularidades históricas. Así, cada una muestra un modelo específico de emergencia, debate y ejecución; por ello es muy difícil que la praxis estatal, realista en las coyunturas económicas y geopolíticas actuales, suceda bajo modelos definidos.

En este sentido, la forma que eligen los autores para poner a prueba el modelo de gradualismo basado en experimentación es novedosa, ya que muestra la complejidad implicada en una política pública y expone algunas características esenciales del caso chino. Las variables retomadas son: la forma que asume la relación política entre tres actores -el gobierno central, los gobiernos locales y las fuerzas del mercado- y el tipo de política pública - distributiva, regulativa y redistributiva.

Orientando. Temas de Asia Oriental. Sociedad, Cultura y Economía. Revista editada por el Centro de Estudios China-Veracruz de la Universidad Veracruzana, México (Centro de Estudios APEC) / año 9 / número 17 / 
Según este razonamiento, la perspectiva de éxito del modelo depende de la forma que asume la dinámica gobierno central-gobiernos locales-mercado. En tanto, las motivaciones para llevar a cabo cambios, experimentos o innovaciones, son distintas para cada uno de estos tres actores; de igual forma la influencia que unos pueden tener sobre otros. Por tanto, su funcionamiento requiere que el gobierno central ceda poder político, a cambio de que los locales se arriesguen y experimenten o innoven.

Dado el modelo altamente competitivo entre las provincias, sobre todo en términos fiscales, las motivaciones de los gobiernos locales están fuertemente emparentadas con la posibilidad de expansión del mercado. (Dussel, 2015) En cambio, el gobierno central debe tener en cuenta el consenso y la legitimidad, por lo que dicha expansión puede ser, en ciertos casos, contraproducente; por ejemplo, cuando genera desigualdad o aumenta brechas regionales.

La dinámica entre estos tres actores puede cambiar. Depende, siguiendo el razonamiento del mismo artículo, del tipo o el área de cada política pública; es decir, del análisis de caso. Los tipos de políticas pueden ser:

a) Sistributivas, aquellas que extienden servicios y recursos públicos a la sociedad -como la construcción de carreteras. Los autores consideran que fueron muy importantes para la expansión del mercado en el país.

b) Regulatorias, en las que el gobierno limita o impone ciertas actividades - como las políticas antimonopolio o de salarios mínimos.

c) Redistributivas, aquellas cuyo fin es mejorar la calidad de vida de la población. En China, estas emergieron para contrarrestar los efectos pro-mercado de las primeras.

El modelo de gradualismo basado en experimentación es más efectivo en el caso de las políticas distributivas, debido a que el gobierno central asume una actitud ambigua, empoderando la autonomía de los ejercicios locales y aceptando que los actores del mercado

Orientando. Temas de Asia Oriental. Sociedad, Cultura y Economía. Revista editada por el Centro de Estudios China-Veracruz de la Universidad Veracruzana, México (Centro de Estudios APEC) / año g / número 17 / 
experimenten en las provincias. Para que esto funcionara, la descentralización fue una condición necesaria.

En el caso de las regulatorias, la dinámica es otra. El central impone su voluntad frente al mercado y a los poderes provinciales a través de sanciones. En este tipo de política, el modelo basado en experimentación no es efectivo, ya que los gobiernos locales tienen todavía margen de maniobra -en términos de negociación o resistencia-, pero no los actores del mercado.

Respecto a las redistributivas, el gobierno central también asume el motor de acción frente a la inactividad del mercado, por tratarse de políticas que suelen ir en detrimento de este último. Las autoridades locales no tienen incentivos para motivar la experimentación como en las anteriores y la presión de la sociedad -en términos de legitimidad o demandaaparece como otro actor relevante.

Por tanto, el modelo de producción de políticas públicas basado en experimentación o gradualismo funciona únicamente cuando la acción del gobierno central es ambigua y los poderes locales tienen margen y motivaciones para arriesgarse a experimentar. De no estar dadas estas condiciones, el modelo de arriba hacia abajo es más efectivo; con la salvedad de que cada análisis de caso complejiza la fórmula, ya que una misma política puede ser distributiva y redistributiva o puede cambiar con el tiempo. Sin embargo, resulta importante volver a resaltar el rol fundamental que tuvo la dinámica centralización-descentralizaciónpara las políticas públicas en China y el lugar activo que tiene el mercado como motivador y productor.

Hasta aquí, este apartado presentó algunos de los aportes de dos de los académicos chinos más reconocidos dentro del campo de estudio de las políticas públicas. El punto de partida fue el modelo de configuración de la agenda de políticas públicas, presentado por Wang. Luego surgieron análisis más complejos del mismo autor donde se incorporan variables históricas, la dinámica centralización-descentralización, los efectos de la acción del mercado y preguntas más amplias acerca de la reforma del Estado en China y el concepto de capacidades estatales. Por último, se sistematizó el artículo donde Mei, Liu y Xue ponen a

Orientando. Temas de Asia Oriental. Sociedad, Cultura y Economía. Revista editada por el Centro de Estudios China-Veracruz de la Universidad Veracruzana, México (Centro de Estudios APEC) / año g / número 17 / 
prueba el modelo de gradualismo basado en experimentación, por ser un abordaje integral que involucra muchos matices.

Con esta primera aproximación a la bibliografía existente sobre estudios de políticas públicas chinas como base, la siguiente sección incorpora más investigaciones de intelectuales chinos y aportes provenientes de otros países del mundo. Por tanto, se tratará el concepto de autoritarismo fragmentado - una visión occidental pionera- y sus posteriores revisiones; también se incluirán los artículos que sostienen la incorporación de actores externos y se profundizará en las conceptualizaciones de Heilmann sobre el rol de la experimentación.

\section{Descentralización y fragmentación}

Hay consenso sobre del rol pionero que tuvo el libro de Lieberthal y Oksenberg (1988) para los estudios acerca de la producción de políticas públicas en China. $\mathrm{Su}$ concepto de "autoritarismo fragmentado" -rectificado unos años después por Liberthal y Lamptonconsidera que las decisiones dentro del Estado están principalmente definidas por la tensión entre las autoridades políticas de los escalafones más altos y los distintos actores que componen la estructura burocrática de sistema estatal chino. Cada iniciativa debe pasar por una serie de unidades diversas - ministerios varios, autoridades locales y cuadros políticos intermedios-, por lo que se produce una fragmentación de la autoridad.

Por tanto, para comprender la complejidad de la producción de políticas deben tenerse en cuenta "las realidades de las prácticas burocráticas que afectan tanto a las elites como a los cimientos básicos del sistema”. (Liberthal, 1992) Esta gama de actores internos del Estado no aparece en las investigaciones de Wang ni de Xue, pero podría encajar perfectamente en la definición que el primero hizo sobre el Estado chino, en tanto "complejo concreto de componentes dispares". (Wang, 1991)

Orientando. Temas de Asia Oriental. Sociedad, Cultura y Economía. Revista editada por el Centro de Estudios China-Veracruz de la Universidad Veracruzana, México (Centro de Estudios APEC) / año g / número 17 / 
Para que la producción de políticas sea efectiva en este marco de fragmentación de la autoridad, los líderes ubicados en el gobierno central deben establecer acuerdos en forma horizontal -ministerios y entidades- y vertical -gobiernos locales. La necesidad de este "proceso consultivo" incrementó, según los autores, luego de la reforma de 1978, debido a la mayor descentralización durante la década de los 80, que otorgó a unidades burocráticas de escalones medios y bajos mayor control sobre recursos fiscales. ${ }^{8}$ Nuevamente la dinámica de centralización-descentralización es significativa en el análisis.

Posteriormente, Mertha (2008) puso a prueba el modelo de autoritarismo fragmentado, en su análisis sobre las políticas hidráulicas. Similar al diagnóstico de Wang (2008), consideró que no alcanza con indagar las contradicciones y tensiones dentro de la estructura burocrática del Estado, sino que también es necesario observar el diálogo con actores externos. Su hipótesis es que la producción de políticas públicas en China tiene una tendencia a la pluralización, ya que incorpora a los medios, las organizaciones no gubernamentales (ONG), así como a activistas individuales, entre otros.

Este autor señala que uno de los limitantes del modelo de Lieberthal y Oksenberg es una definición estática. En esta línea, es posible observar cambios al analizar en forma histórica cómo se definieron y ejecutaron las políticas en los distintos períodos. Dicha incorporación de actores no disminuye la fragmentación, pero sí las características autoritarias; de allí que propone llamar a su modelo "autoritarismo fragmentado 2.0".

Según Ma y Lin, previamente citados, uno de los puntos de cambio para esta área de estudio fueron las reformas iniciadas en 2003 en el Río $\mathrm{Nu}$, provincia de Yunnan. Han, Swedlow y Unger (2014) analizan cómo este acontecimiento da por tierra el concepto de autoritarismo fragmentado. Consideran que, a pesar de que el Estado actuó de manera unificada, la oposición de los medios y de las ONG creó una gran tensión en la ejecución de la política. Su artículo utiliza el modelo de coaliciones promotoras, introducido por Sabatier y

\footnotetext{
${ }^{8}$ La cercanía entre las publicaciones de Wang (1991) y Liberthal (1992) da cuenta de cómo la descentralización, principalmente en las políticas fiscales, fue uno de los ejes de discusión más importante de. principios de la década de los 90, con el proceso transicional de reforma del Estado chino. Muchos de los artículos de Wang pueden ser pensados con esa lógica: como documentos que expresan discusiones de época.
}

Orientando. Temas de Asia Oriental. Sociedad, Cultura y Economía. Revista editada por el Centro de Estudios China-Veracruz de la Universidad Veracruzana, México (Centro de Estudios APEC) / año g / número 17 / 
Weible (2014), que analiza cómo distintos actores construyen coaliciones para promover cambios en políticas, a partir de determinadas creencias compartidas.

De igual forma, Heilmann utiliza el modelo de coaliciones promotoras en coautoría con a Shih (2013). Allí deja a un lado su análisis sobre la experimentación y presenta las diferentes facciones que disputaron y condujeron los planes nacionales de políticas industriales desde 1978 hasta 2012. Por tanto, la variable que define la producción de políticas públicas presenta un mapa sobre las alianzas que lideraron la toma de decisiones, organizadas por su orientación respecto a la política económica.

Las cuatro orientaciones que tipifica este autor son: los grupos a favor de la liberalización del mercado, los interesados por la política industrial, la planificación estatal orientada y la planificación estatal imperativa. Un último aspecto novedoso de este análisis de caso es que describe a detalle la influencia del modelo japonés; de esta manera presenta un ejemplo donde la influencia externa es un actor fundamental.

Otra publicación notable de Heilmann es su libro sobre el sistema político chino (2017), donde define la producción de políticas públicas como plausible de tener resultados inciertos y estar impulsada por intereses, interacciones y conflictos. Esta definición dinámica alude a una retroalimentación continua y afirma que la formulación de políticas no está predeterminada de manera directa por la historia ni por el tipo de régimen o instituciones.

Este autor separa su modelo de experimentación en políticas en tres etapas: primero define puntos o zonas experimentales locales; después selecciona modelos experimentales exitosos desde el punto hacia a la superficie, revisando la posibilidad de generalizar o no el caso; por último evalúa las políticas exhaustivamente, antes de ser implementadas en las legislaciones nacionales. Argumenta que este último proceso puede durar muchos años.

También distingue otras características específicas del caso chino. Por ejemplo, que en los momentos de crisis el gobierno tiene la capacidad de cambiar de un sistema más

Orientando. Temas de Asia Oriental. Sociedad, Cultura y Economía. Revista editada por el Centro de Estudios China-Veracruz de la Universidad Veracruzana, México (Centro de Estudios APEC) / año g / número 17 / 
fragmentado a uno más centralizado y de mayor concentración de poder. Heilmann incorpora elementos más laxos, como la posibilidad de experimentación o innovación, y más duros, como la planificación estratégica y los procesos a mediano y largo plazo.

En los párrafos anteriores se recapitulan investigaciones que dan cuenta de las consecuencias de la descentralización en términos de mayor diálogo del gobierno central con actores internos y externos. Esta caracterización debe ser vista en clave histórica, ya que hace referencia a la forma en que se asumió el proceso de reforma del Estado chino en las primeras dos décadas posteriores; los artículos de Wang sobre la involución de las capacidades estatales iban en esa dirección.

No obstante, existe otra serie de investigaciones posteriores al 2003 que vuelven a la hipótesis de la centralización. A un rol más sólido y propositivo por parte de los principales líderes del gobierno central y a la vigencia del concepto de autoritarismo fragmentado. Por tanto, el siguiente apartado presenta un diálogo entre esta postura y las que continúan en línea con la tendencia a la participación.

\section{Cambios del siglo XXI: ¿participación, centralismo o ambas?}

Resulta cada vez más evidente que la literatura existente acerca de los distintos modelos y cualidades de la producción de políticas públicas cambió con el tiempo. El Estado en China, su orientación, su forma de administración y modelo de desarrollo no fue igual en la década de los 80 que en los 90. Lo mismo sucede con sus políticas públicas. Para comprender las particularidades de cada momento, es determinante observar las características de la dinámica de centralización-descentralización.

Como afirman varios autores -Ngok, Huang, Ma, Zhang, Gao, Yang y Li, por nombrar algunos-, 2003 fue un año de transformación para el Estado Chino. Uno de estos cambios fue la nueva tendencia al fortalecimiento del poder político de los líderes principales del gobierno central. Este momento se traduce en el estudio sobre políticas públicas en debates entre la 
tendencia a la mayor participación y los efectos de la nueva centralización. Pero, ¿pueden los modelos de experimentación e innovación coexistir con menos margen de maniobra para gobiernos locales y actores externos?, ¿es imposible que la centralización conviva con la participación?

Chen y Naughton (2013) cuestionan esta tendencia a la incorporación de actores y a la innovación. En sus análisis sobre los cambios en las políticas tecno-industriales afirman que la producción de políticas públicas adquirió en 2003 características cercanas al modelo de arriba hacia abajo. Incluso discuten la fragmentación, ya que observan una hegemonía de los líderes políticos más importantes frente a las unidades burocráticas. También aseguran que los períodos en los que la descentralización o la ambigüedad eran motores de la producción de políticas resultaron mucho menos eficaces que los de centralización.

En su artículo, sostienen que a partir de ese año hay una institucionalización de la producción de políticas con distintas fases. La primera corresponde al momento de "gestación de políticas", en el que distintos actores descentralizados presentan posibles políticas en respuesta a una agenda general establecida previamente por los principales líderes -este momento incluye grados de pluralidad. Luego inicia la fase en la que los líderes políticos se dedican a la "formulación de políticas pragmáticas", que suele materializarse en un documento oficial. Según los autores, la cúpula de gobierno "estructura el proceso de consulta y manipula esa estructura para lograr los resultados que prefiere”. (Chen \& Naughton, 2013)

Con ese documento como punto de partida, inicia la fase de "especificación de la política". A medida que la política se prepara para su implementación, distintos grupos de intereses luchan por moldearla. A partir de esta disputa se definen cuestiones como la jurisdicción o el control de los recursos; en este momento de especificación, las distintas unidades burocráticas adquieren un margen de maniobra.

Orientando. Temas de Asia Oriental. Sociedad, Cultura y Economía. Revista editada por el Centro de Estudios China-Veracruz de la Universidad Veracruzana, México (Centro de Estudios APEC) / año g / número 17 / 
La cuarta y última fase es la de "implementación de la política", en la que se ejecutan las actividades pertinentes. Este momento concluyente tiene en cuenta también los resultados, en términos de éxito, éxito imperfecto o consecuencias imprevistas.

Por lo tanto, los autores se acercan al concepto de autoritarismo fragmentado o proponen un regreso a este, con menos grados de fragmentación. Otros, en cambio, apoyan la hipótesis de la tendencia al aumento de participación de Wang; allí se encuentran, por ejemplo, Wang y Zhang (2018), quienes sostienen que China pasó por una transición desde un modelo gerencial a un modelo participativo. Llegan a esta conclusión tras analizar casos de políticas en las que gobiernos locales -como Xiamen, Shanghái, Chonqing y Hunan- incorporaron actores no estatales al proceso de toma de decisiones.

El modelo gerencial, estaba dirigido en forma vertical por los órganos del Estado-partido, organizados en forma piramidal y burocrática. Las decisiones de los líderes del Partido Comunista Chino de acelerar la transición a una economía de mercado minaron su efectividad. En este caso, la descentralización no aparece como una decisión del gobierno central, sino como efecto de la mayor presencia de actores del mercado.

En contraposición, el modelo participativo otorga mayor peso a los intereses de los ciudadanos a la hora de influir en la agenda de las políticas; por tanto, las reuniones de consulta y negociación comienzan a ser canales de comunicación cada vez más utilizados. Un sistema abierto de información y una mayor representación colectiva de intereses son los dos componentes institucionales que favorecen esta incorporación de actores.

En este aspecto, los Think tanks son actores principales a la hora de nombrar los procesos más abiertos de producción de políticas públicas. Según Ma y Lin (2012), estos pueden pertenecer al gobierno, empresas, universidades o estar manejados por miembros de la sociedad civil. En tanto, Köllner, Zhu y Abb (2018) los definen como “organizaciones cuya principal misión es informar e influir en políticas públicas a partir de investigaciones y análisis”, y explican que en los últimos años hubo un aumento cuantitativo en el Este de Asia.

Orientando. Temas de Asia Oriental. Sociedad, Cultura y Economía. Revista editada por el Centro de Estudios China-Veracruz de la Universidad Veracruzana, México (Centro de Estudios APEC) / año 9 / número 17 / 
En el caso de China, resalta un especial reconocimiento de líderes políticos contemporáneos sobre el valor de estas organizaciones, pues asocian su crecimiento con el aumento del rol del mercado en la economía y la emergencia de profesionales e intelectuales en el ámbito; así como con el regreso de estudiantes desde el extranjero. Una reciente materialización del cambio en el discurso oficial acerca de su influencia puede verse en el reporte del gobierno central de 2013 y la promoción de nuevos tipos de Think tanks con características chinas.

Otro artículo que no coincide con los modelos de mayor control por parte del gobierno central, es el de Zhu y Xiao (2015) sobre los procesos de innovación política. Lo novedoso es que critican aquellos análisis que toman a los gobiernos locales como una totalidad sin divergencias y ponen el eje en los agentes fundamentales para la innovación. Por eso, analizan el rol de los "políticos emprendedores".

Hasta el momento, los únicos que habían incorporado agentes en sus modelos de producción política habían sido Lieberthal y Oksenberg (1988), al nombrar el papel que jugaban los líderes más importantes del gobierno en la toma de decisiones. En este caso, la referencia es sobre aquellas personas que movilizan recursos, establecen redes sociales y canales de comunicación eficaces y crean las condiciones de posibilidad para la innovación política desde los gobiernos locales.

El modelo de innovación política propuesto por Zhu y Xiao está basado en tres etapas: la conceptualización de la idea, el diseño del programa de la política y la implementación. En línea con las investigaciones de Heilmann, se ubica la descentralización y la promoción de competitividad entre gobiernos locales. Para evaluar estos factores, los investigadores analizan el caso del establecimiento del seguro de salud en la ciudad de Nanguo, al sudeste de China, desde las primeras reformas en 1992 hasta su implementación en 2008.

Estas posturas parecen, en un primer momento, contradictorias. Pero, ¿puede un modelo de producción de políticas públicas concentrar poder en pocas manos y al mismo tiempo

Orientando. Temas de Asia Oriental. Sociedad, Cultura y Economía. Revista editada por el Centro de Estudios China-Veracruz de la Universidad Veracruzana, México (Centro de Estudios APEC) / año 9 / número 17 / 
volverse más participativo?, ¿puede haber incorporación de actores de la ciudadanía al proceso de toma de decisiones, estando manipulado por una cúpula del Gobierno?

Es erróneo definir un único modelo de producción de políticas públicas en China desde 1978 hasta la actualidad. Primero, porque es posible que coincidan varios modelos al mismo tiempo. Aquí, los aportes de Mei, Liu y Xue (2012) cobran relevancia; no solo la dinámica descentralización-centralización explica el modelo de producción de políticas, sino también el tipo de política.

Que en la reforma de salud de Nanguo la calve hayan sido agentes internos del gobierno local o que las organizaciones ambientalistas hayan tenido un rol activo para la construcción del tren magnético elevado de Shanghái, no implica que el gobierno central no pueda encabezar el área de políticas tecno-industriales. (Chen y Naughton, 2013)

No obstante, la convivencia de varios modelos, según el área de la política que se trate, constituye la efectividad de la praxis estatal. En algunos casos es estratégico que los planes nacionales se ejerzan sin fisuras, por lo que hay una decisión política de mayor verticalidad y control. En otros, la incorporación de actores de la ciudadanía, o la extensión del margen de acción de los gobiernos locales permite un consenso.

A esto se dedica el artículo de Xue y el grupo de Tsinghua, mencionado con anterioridad: a examinar el modelo de gradualismo basado en experimentación según políticas distributivas, redistributivas o regulativas. De manera que los análisis de caso son igual de importantes que el contraste de los resultados.

Por otra parte, el Estado, y el PCC atravesaron un proceso de aprendizaje cuyo resultado es, justamente, la convivencia de varios modelos. Esto sucedió gracias al carácter transicional del proceso. (Qian, 2002) En este sentido, no hay momentos suma-cero de cambios, sino que se fueron incorporando aprendizajes en la medida en que el país transitó su reforma. Por tanto, el siguiente apartado introduce la variable temporal y la idea de reforma transicional del Estado.

Orientando. Temas de Asia Oriental. Sociedad, Cultura y Economía. Revista editada por el Centro de Estudios China-Veracruz de la Universidad Veracruzana, México (Centro de Estudios APEC) / año 9 / número 17 / 


\title{
6. Transición y aprendizaje
}

\author{
"El sentido histórico escapará a la metafísica para convertirse en el \\ instrumento privilegiado de la genealogía si no se posa sobre ningún absoluto. \\ No debe ser más que esta agudeza de una mirada que distingue, reparte, \\ dispersa, deja jugar las separaciones y los márgenes una especie de mirada \\ disociante, capaz de disociarse a sí misma y de borrar la unidad de este ser \\ humano que se supone conducirla soberanamente hacia su pasado" \\ -M. Foucault.
}

Luego de 70 años de dirección de una nación y poco más de 40 de reforma continua, el PCC contiene en su haber el estigma de los sucesos pasados. Estigma, entendido como lo hace Foucault, en tanto marcas que dejan las acciones y contradicciones del paso del tiempo. En este caso, la capacidad del Estado en China avanzó en su desarrollo histórico, acumulando aprendizajes.

En esta trayectoria, desdibujó praxis emblemáticas: mantuvo constantes algunas decisiones estratégicas -incluso frente a fuertes presiones geopolíticas-, incorporó dispersiones y dio lugar a la creatividad del ejercicio estatal -apremiando aquellas que funcionaron. Los distintos modelos de producción de políticas públicas en China no emergieron como reemplazo de los anteriores, sino en convivencia.

Kinglun Ngok es un académico importante para la literatura existente en este campo. En su trabajo, es posible encontrar investigaciones acerca de la seguridad social -Ngok, 2010-, los efectos de la dinámica centralización-descentralización en políticas educativas -Ngok y Chan, 2004- y una serie de artículos que hacen referencia a los cambios en el paradigma de las políticas -Ngok y Huang, 2014.

Según el autor, en concordancia los temas presentados en el apartado anterior, 2003 significó un año de transformaciones. La administración de Hu Jintao y Wen Jiabao

Orientando. Temas de Asia Oriental. Sociedad, Cultura y Economía. Revista editada por el Centro de Estudios China-Veracruz de la Universidad Veracruzana, México (Centro de Estudios APEC) / año g / número 17 / 
pronunciaron un nuevo marco para todas las acciones estatales, llamado "teoría del desarrollo científico", contrapuesto al que guio al Estado desde la reforma hasta el inicio del nuevo siglo.

Ngok (2009) explica el cambio de paradigma en términos de aprendizaje en políticas. Muestra un modelo en el que entrecruza la capacidad estatal -que puede ser alta o baja-con los actores dominantes del subsistema de la política - de la sociedad o del Estado. La primera variable es definida como la capacidad del Estado de movilizar consentimiento social y económico para alcanzar determinados objetivos públicos y tiene un componente intrínseco, pues asume al Estado como actor estratégico que formula y aplica políticas públicas de manera coherente; así como uno extrínseco, relacionado con la capacidad de definir estándares, monitorear el desempeño e influenciar la dirección de las inversiones de firmas privadas a cambio de subsidios.

El modelo de aprendizaje en políticas está descrito de la siguiente manera: si la capacidad estatal es alta y el actor que determina el subsistema político proviene de la sociedad, el tipo de aprendizaje es "social"; si proviene del Estado es “instrumental". Cuando, en cambio, la primera variable es baja y dominan actores de la sociedad, el aprendizaje es "nulo" y si son actores estatales, es "limitado".

Esta tipología es planteada para explicar el cambio de paradigma del 2003. Pero, al estar pensado en términos de aprendizaje, brinda una forma novedosa de ver los cambios en la producción de políticas públicas, distinta a las concepciones de suma-cero entre un modelo y otro. Por ello, a la hora de problematizar la reforma del Estado en China es más acertado hablar en términos de transiciones que de cambios.

En este ámbito, la investigación de Qian y su concepto de instituciones transicionales, adquiere relevancia analítica. El académico postula que el análisis del cambio chino generalmente se concentra en sus resultados, pero que esto no refleja cómo funcionó la reforma; de esta forma, introduce en contraposición el concepto de instituciones transicionales. Para mayor análisis sobre este concepto, la tesis doctoral de Tejeda Canobbio es esclarecedora:

Orientando. Temas de Asia Oriental. Sociedad, Cultura y Economía. Revista editada por el Centro de Estudios China-Veracruz de la Universidad Veracruzana, México (Centro de Estudios APEC) / año 9 / número 17 / 
El concepto de institución transicional rechaza el individualismo y asume la primacía analítica de la política y sus medios para constituir los mercados, reconociendo una prioridad causal y temporal entre instituciones e individuos, sin asumir que estos limitan su comportamiento ni que sus motivaciones son fijas. Así pues, la existencia y funcionamiento de las instituciones transicionales se aleja de la perspectiva neoclásica o incluso de los neo institucionalistas más ortodoxos, al embarcarse hacia otra dirección teórica en la que la historia y la cultura juegan un papel muy importante. (Tejeda, 2009)

Este enfoque es útil para considerar no solo la reforma institucional, sino también una reforma estatal más general, porque incorpora el tiempo como una variable importante y da lugar a errores, resistencias, máscaras y consecuencias inadvertidas. En palabras del autor, "hacer que las instituciones estén bien es un proceso que involucra incesantes cambios que interactúan con las condiciones iniciales”. (Qian, 2002)

Qian analiza cinco medidas implementadas para desarrollar una economía de mercado: el proceso de dos vías para la liberalización de precios y cuotas, la creación de empresas de pueblos y aldea -TVE, por sus siglas en inglés--, la descentralización fiscal, la banca anónima y la reforma de empresas estatales.

Pero más que las discusiones sobre la reforma económica, interesa para este apartado la concepción sobre las instituciones "imperfectas" o fuera de lo establecido, considerada clave para el éxito del caso chino. Así lo explica el experto:

Las reformas institucionales exitosas en economías en desarrollo generalmente no son una copia fiel de las instituciones típicas de una economía desarrollada. No requieren serlo y a veces no deberían serlo. No requieren serlo porque en esas economías existe todavía espacio para mejorar la eficiencia que no implica ajustes detallados prefabricados al principio. No deberían serlo porque las condiciones iniciales y específicas del país requieren de arreglos contextuales especiales. Así, las instituciones

Orientando. Temas de Asia Oriental. Sociedad, Cultura y Economía. Revista editada por el Centro de Estudios China-Veracruz de la Universidad Veracruzana, México (Centro de Estudios APEC) / año g / número 17 / 
transicionales reflejan una variedad de formas no estandarizadas, pero efectivas para dirigir el cambio institucional. (Íbid.)

El concepto de lo "transicional" presenta similitudes con los modelos de experimentación, innovación o gradualismo ya explicados, pero le da mayor entidad a la variable temporal y refleja rasgos comunes en el caso chino. Algo similar sucede con la producción de políticas públicas.

A pesar de que con el cambio de liderazgo del 2003 existió una merma en las dinámicas de mayor margen para actores externos al gobierno central, los modelos de ejecución de reformas, toma de decisiones e implementación, continúan siendo novedosos, heterodoxos y heterogéneos. Por tanto, el aprendizaje no cesa y la transición tampoco.

\section{Conclusiones}

Este artículo recapituló los principales debates de la literatura existente acerca del área de estudios sobre políticas públicas chinas. En este repaso, se encuentran fundamentos de la sociología china -en tanto posible especialización- y estudios acerca del papel del Estado, principalmente a partir de su reforma en1978.

En este sentido, se presentaron primero dos autores centrales: Wang Shaoguang y Xue Lan, con la intención de darle jerarquía a académicos locales y sus conceptualizaciones. Así como de reflejar algunos de los debates que transcurrían acerca del Estado en los distintos períodos.

La definición de "sistema estatal" y la prioridad del concepto de capacidad estatal para comprender muchas de las reformas, son dos de los puntos destacables de los documentos recuperados de la producción de Wang. Por otra parte, la puesta a prueba del modelo de gradualismo basado en experimentación, realizado por Xue en coautoría con Liu y Mei, es una de las investigaciones más importantes sobre políticas públicas.

Orientando. Temas de Asia Oriental. Sociedad, Cultura y Economía. Revista editada por el Centro de Estudios China-Veracruz de la Universidad Veracruzana, México (Centro de Estudios APEC) / año g / número 17 / 
Este trabajo muestra la convivencia de varios modelos al mismo tiempo, explicita el rol clave que juega la interrelación entre el gobierno central, los gobiernos locales y el mercado e invierte el eje del análisis, ubicando al tipo de política pública como variable independiente para el proceso de debate y ejecución. Esta propuesta, por su complejidad e integralidad, debe ser considerada seriamente a la hora de un próximo estudio de caso.

Posteriormente, este artículo enumeró otra serie de autores y textos que circulan bajo el actualmente denominado "período de fragmentación de las políticas públicas". Desde la reforma de 1978 hasta principios de los 90, la descentralización fue una de las variables fundamentales para comprender muchas de las transformaciones estructurales de la transición china. Por tanto, fragmentación puede entenderse en un sentido productivo y creador, pero también puede significar un riesgo en términos de capacidades estatales.

En este sentido, la década de los 90 incorpora otros matices. La experimentación y el rol de los gobiernos locales continuaron manteniendo su peso específico, pero algunas medidas como la recentralización fiscal de 1994 o las reformas administrativas de 1998 implicaron diferencias relevantes respecto a la década anterior. No obstante, el modelo de gradualismo basado en experimentación es hijo de esos tiempos; por ello, el segundo apartado de este documento incluyó algunas de las discusiones al respecto.

Luego se describieron algunas de las posturas sobre los modelos de producción de políticas públicas a partir del cambio de liderazgo de 2003. Se consideraron representativas de este momento las discusiones entre quienes sostenían la hipótesis de una tendencia al crecimiento de la participación, contra aquellos que demostraron un proceso de recentralización.

Por último, se presentó el concepto de instituciones transicionales, acuñado por Qian, en cuanto clave para una comprensión del caso chino. Primero, con sentido histórico -teniendo en cuenta sus contradicciones, dispersiones lejos de las categorías estáticas y absolutas-; segundo, conforme a sus particularidades: instituciones, políticas y decisiones 
gubernamentales alejadas de sus concepciones ortodoxas - una praxis estatal menos rígida de lo que suele considerarse.

La decisión, en este caso, se centró en las discusiones acerca de la producción de políticas públicas; en el momento creativo - la praxis- más que el resultado. Poner el eje en este punto puede arrojar reflexiones significativas para desentrañar aún más el proceso de reforma del Estado en China.

En este recorrido conceptual, una de las reflexiones primigenias fue la dinámica centralización-descentralización como uno de los puntos independientes más importantes, ya que sin su comprensión de manera integral y longitudinal no es posible hilar fino en los detalles del proceso de reforma chino. De esta forma, considerar el sentido histórico junto con el concepto de lo transicional permite comprender la existencia de distintos modelos, junto con sus transformaciones y convivencias heterogéneas.

En suma, desde la reforma de 1978 hasta la actualidad existieron distintos tipos ideales de modelos de producción de políticas públicas:

1. gradualismo basado en experimentación, a través del margen de acción cedido a los gobiernos locales;

2. innovación por presión de actores externos al Estado-Think Tanks, ONG, participación social, etc., y

3. centralidad estratégica, ejecutada por los líderes del gobierno central, considerando sus matices e interrelaciones.

Según el período histórico, algunos tuvieron más relevancia que otros o se utilizaron para áreas de mayor importancia. Durante la década de los 80, se optó por el modelo de gradualismo basado en experimentación para romper con las unidades del maoísmo y permitir el ingreso de actores del mercado.

Entre 1989 y 1991, las coaliciones que todavía abogaban por modelos de planificación estatal centralizada tuvieron un intento de liderazgo que rápidamente resultó fallido. La

Orientando. Temas de Asia Oriental. Sociedad, Cultura y Economía. Revista editada por el Centro de Estudios China-Veracruz de la Universidad Veracruzana, México (Centro de Estudios APEC) / año 9 / número 17 / 
reforma era una fuerza, contradictoria, pero imparable. En los tempranos 90, comenzó la convivencia entre el modelo de gradualismo basado en experimentación junto con el de centralidad estratégica. A fines de la década, la participación de actores externos emergió, producto de los efectos de la liberalización de la economía, dando lugar a una aparición tímida del modelo de innovación por presión de actores externos al Estado. A partir de 2003, la centralidad estratégica cobró mayor relevancia, pero no eliminó por completo la experimentación ni la participación de actores de la sociedad, sino que los mantuvo.

La reforma de Estado en China, desde 1978, fue y continúa siendo compleja, pero muy novedosa; pero las simplificaciones que la estudian únicamente a partir de medidas económicas pierden de vista la riqueza de la variable política. Por tanto, entender en profundidad el desarrollo de la capacidad estatal de China a lo largo del tiempo puede ser útil para muchas de las preguntas que ocupan la agenda de los Estados a nivel mundial.

\section{Bibliografía}

Chen Ling \& Naughton, B. (2013). The Emergence of Chinese Techno-Industrial Policy: From Megaprojects to Strategic Emerging Industries, 2003-2011.

Dussel, E. (2015) “The Omnipresent Role of China's Public Sector in Its Relationship with Latin America and the Caribbean”. In Dussel, E. \& Armony, A. (Coords.). Beyond Raw Materials Who are the Actors in the Latin America and Caribbean-China Relationship? Buenos Aires: Friedrich-Ebert-Stiftung, Red Académica de América Latina y el Caribe sobre China, Center of Latin American Studies/University of Pittsburgh, Fundación Foro Nueva Sociedad.

Orientando. Temas de Asia Oriental. Sociedad, Cultura y Economía. Revista editada por el Centro de Estudios China-Veracruz de la Universidad Veracruzana, México (Centro de Estudios APEC) / año g / número 17 / 
Foucault, M. (1977). "Nietzsche, Genealogy, History.” In Bouchard, D. (Ed.). Language, Counter-Memory, Practice: Selected Essays and Interviews. Ithaca: Cornell University Press.

García, Á. (2009). “El papel del Estado en el modelo productivo nacional”. Ponencia presentada en el seminario Organización Económica en la nueva Constitución Política del Estado, realizado por el Viceministerio de Presupuesto y Contaduría del Ministerio de Economía y Finanzas Públicas, en el auditorio del Banco Central de Bolivia, La Paz.

Han, H., Swedlow, B. \& Danny, U. (2014). "Policy Advocacy Coalitions as Causes of Policy Change in China? Analyzing Evidence from Contemporary Environmental Politics”. In Journal of Comparative Policy Analysis: Research and Practice.

Heilmann, S. (2007). "Policy Experimentation in China's Economic Rise". In Studies in Comparative International Development(43). N. 1, pp. 1-26.

(2017). China's Political System. Rowman \& Littlefield Publishing Group, Inc.

Heilmann, S. \& Shih, L. (2013). The Rise of Industrial Policy in China, 1978-2012. HarvardYenching Institute Working Paper Series.

Kroeber, A. (2016). China's economy what everyone needs to know. US: Oxford University Press.

Köllner, P., Zhu Xufeng \& Abb, P. (2018). "Understanding the Development of Think Tanks in Mainland China, Taiwan, and Japan”. In Pacific Affairs(91), pp. 5-26.

Lieberthal, K. \& Lampton, D. (1992). Bureaucracy, Politics, and Decision Making in PostMao China. Los Angeles: University of California Press.

Orientando. Temas de Asia Oriental. Sociedad, Cultura y Economía. Revista editada por el Centro de Estudios China-Veracruz de la Universidad Veracruzana, México (Centro de Estudios APEC) / año g / número 17 / 
Lieberthal, K. \& Oksenberg, M. (1988). Policy Making in China. Leaders, Structures and Processes. Princeton, New Jersey: Princeton University Press.

Li, Shi. (2011). "Issues and Options for Social Security Reform in China". In China: An International Journal(09), N. 01, pp. 72-109.

Liping, Sun (2008). "Societal Transition New Issues in the Field of the Sociology of Development”. In Modern China(34). N. 1. Sage Publications

Ma Jun \& Lin. (2012). "Policymaking in China: A review of Chinese Scholarship". In The China Review. An Interdisciplinary Journal on Greater China(12), N. 1.

Mei Ciqi, Liu Zhilin \& Xue Lan. (2012). "When gradualism might fail? Politics of Policy Experiment in China”. School of Public Policy \& Management, Tsinghua University, Beijing, China. Paper Presented at the 22rd International Political Science Association World Congress. Madrid, Spain, July 8-12.

Mertha, A. (2008). China's water warriors: citizen action and policy change. Cornell University Press.

Ngok, K. (2010). "Social Assistance Policy and Its Impact on Social Development in China: The Case of the Minimum Living Standard Scheme (MLSS)". In China Journal of Social Work.

(2009) "State capacity, Policy learning, and Policy Paradigm Shift: Institutionalization of the Theory of Scientific Development in China". In The Korean Journal of Policy Studies( 24). N. 2. Seoul National University, pp. 1-23

Ngok, K. \& Chan Kin Keung, D. (2004). "Towards Centralization and Decentralization in Educational Development in China: The Case of Shanghai”. In M. Ka-Ho (Ed.). 
Centralization and Decentralization. Kluwer Academic Publishers and Comparative Education.

Ngok, K. \& Huang, G. (2014). "Policy Paradigm Shift and the Changing Role of the State: The Development of Social Policy in China since 2003”. In Social Policy \& Society. Cambridge University Press.

O’Donnell, G. (S.F.) “Acerca del Estado en América Latina contemporánea. Diez tesis para discusión". Texto preparado para el proyecto "La Democracia en América Latina," propiciado por la Dirección para América Latina y el Caribe del Programa de las Naciones Unidas para el Desarrollo (DRALC-PNUD). Disponible en http://www.unsa.edu.ar/histocat/instituciones/odonnell.pdf.

Qian, Yingyi. (2002). How Reform Worked in China. Department of Economics. University of California, Berkeley. Berkeley, CA 94720-3880. yqian@econ.berkeley.edu.

Skocpol, T. (1995). "El Estado regresa al primer plano: Estrategias de análisis en la investigación actual”. En: Grompone, R. (Ed.); Adrianzén, A.; Cotler, J. \& Sinesio, L. (Comps.). Instituciones políticas y sociedad. Lecturas introductorias. Lima: IEP, pp. 92-129.

Tejeda, E. (2009). La propiedad como una institución transicional: china y la industria electrónica de 1980 a 2005. Tesis doctoral. Universidad Nacional Autónoma de México.

Wang Shaoguang. (1991). From Revolution to Involution: State Capacity, Local Power, and [Un] governability in China. Yale University.

(1994). The Compatibility of Public Ownership and the Market Economy: A Great Debate in China.

Orientando. Temas de Asia Oriental. Sociedad, Cultura y Economía. Revista editada por el Centro de Estudios China-Veracruz de la Universidad Veracruzana, México (Centro de Estudios APEC) / año g / número 17 / 
(1995). "Learning by Debating: The Changing Role of the State in China's Economy and Economics Theories". In Policy Studies Journal(23). N. 1, pp. 11-25.

(1995). "China pension provision and pension administration". A background paper for the World Bank project China: Pension System Reform. China and Mongolia Department, East Asia and Pacific Regional Office, the World Bank.

(1995). "The Rise of the Regions: Fiscal Reform and the Decline of Central State Capacity in China". In Walder, A. (Ed.). The Waning of the Communist State: Economic Origins of Political Decline in China and Hungary. Berkeley: University of California Press, pp. 87-113.

(2000). The Changing Role of Government. Department of Political Science Yale University.

(2010). China's double movement in health care(46).

Wang Xixin \& Zhang Yongle. (2018). The Rise of Participatory Governance in China: Empirical Models, Theoretical Framework and Institutional Analysis. Penn Law: Legal Scholarship Repository.

Weible, C. \& Sabatier, P. (2014). "A Guide to the advocacy Coaliton Framework". In Theories of the policy process. Westview Press, a member of the Persus Books Group.

Whiting, S. (2000). Power and Wealth in Rural China. The Political Economy of Institutional Change. University of Washington.

$\mathrm{Xu}, \mathrm{C}$. (2011). "The fundamental institutions of China's reforms and development". In Journal Of Economic Literature(49), N. 4, pp. 1076-1151.

Orientando. Temas de Asia Oriental. Sociedad, Cultura y Economía. Revista editada por el Centro de Estudios China-Veracruz de la Universidad Veracruzana, México (Centro de Estudios APEC) / año g / número 17 / 
Xue Lan \& Kuotsai, Tom Liou. (2012). “Government Reform in China Concepts and Reform Cases”. In Review of Public Personnel Administration 32(2). SAGE Publications, pp. $115-133$.

Zavaleta, R. (1990). EI Estado en América Latina. Col. Obras completas, T. 3. La Paz: Editorial Los Amigos del Libro.

Zhu Yapeng \& Xiao Diwen. (2015). "Policy entrepreneur and social policy innovation in China”. In The Journal of Chinese Sociology.

Orientando. Temas de Asia Oriental. Sociedad, Cultura y Economía. Revista editada por el Centro de Estudios China-Veracruz de la Universidad Veracruzana, México (Centro de Estudios APEC) / año g / número 17 / 